\title{
ANÁLISIS DE LOS PROCEDIMIENTOS Y CRITERIOS UTILIZADOS POR ORGANISMOS EUROPEOS NACIONALES Y MULTILATERALES PARA LA EVALUACIÓN Y SEGUIMIENTO DE PROYECTOS DE INVESTIGACIÓN EDUCATIVA
}

\author{
ANALYSIS OF THE PROCEDURES AND CRITERIA USED BY NATIONAL \\ AND MULTILATERAL EUROPEAN INSTITUTIONS FOR THE EVALUATION \\ AND FOLLOW-UP OF EDUCATIONALRESEARCH PROJECTS
}

\author{
Consuelo Vélaz de Medrano Ureta*, Nuria Manzano Soto** y \\ Belén Fernández D'Andrea***
}

\begin{abstract}
RESUMEN
El artículo presenta el proceso y resultados obtenidos en esta investigación cuyo objetivo era analizar los procesos y criterios utilizados por distintos organismos europeos para valorar los proyectos de investigación educativa presentados a las convocatorias públicas y el tipo de seguimiento utilizado para controlar los avances y calidad de los proyectos que se financiaron. El trabajo de recogida de datos se centró en las instituciones de investigación educativa más representativas de Inglaterra (National Fundation for Educational Research, NFER), de Francia (Institut National de Recherche Pédagogique, INRP) y de la Comisión Europea (a través de todos los programas de investigación educativa). Los resultados obtenidos se centran en un análisis de contenido que nos permitió extraer los criterios utilizados más profusamente para valorar la calidad de los proyectos de investigación educativa.
\end{abstract}

Palabras clave: Investigación educativa, criterios de evaluación, unión europea, convocatorias de investigación.

\section{ABSTRACT}

The article presents the process and results obtained in this research, which aims to analyse the processes and criteria used by different European organisms to weight educational research projects pre-

* Consuelo Vélaz de Medrano, es Profesora Titular de Orientación e Intervención Psicopedagógica. UNED

** Nuria Manzano Soto es Doctora en Ciencias de la Educación y Profesora en la Facultad de Educación. UNED (correo electrónico: nmanzano@edu.uned.es).

*** Belén Fernández D’andrea, es Licenciada en Pedagogía y orientadora en un Equipo de Orientación Educativa y Psicopedagógica en la Comunidad de Madrid. 
sented for public grants, and the type of follow up used to control the progress and quality of the projects that got financial support. The data gathering process focussed in the educational research institutions more representatives in England (National Foundation for Educational Research, NFER), de Francia (Institut National de Reserche Pédagogique, INRP) and the European Comission (through all educational research programs). Results obtained focus in a content analyses that allows us extract the criteria more widely used to weight the quality of educational research projects.

Key words: Educational research, research criteria, European Union, research grants.

\section{Introducción}

La investigación que se presenta se realizó por encargo del Centro de Investigación y Documentación Educativa (CIDE) en el año 2001. El objetivo general del estudio fue "conocer, analizar y valorar los procesos y criterios utilizados por distintos organismos europeos para la evaluación y seguimiento de proyectos de investigación educativa". Dicho objetivo está referido tanto a los programas que lleva a cabo la Comisión Europea, como a los que emplean algunos de los principales organismos nacionales que promueven o financian investigación educativa, como es el caso de Francia e Inglaterra, entre otros. Para ello se comenzó acometiendo dos tareas:

a) La identificación —orientada por el CIDE — de instituciones europeas de prestigio, responsables de la coordinación, promoción y apoyo a la investigación educativa

b) La elaboración y envío de un cuestionario dirigido a las instituciones seleccionadas - la Comisión Europea, la National Fundation for Educational Research (NFER) y el Institut National de Recherche Pédagogique (INRP). Este cuestionario permitía afinar algunas cuestiones sobre las prioridades del organismo en materia de investigación educativa, el tipo de convocatorias que ofertan, los grupos o equipos a los que van destinadas esas ayudas, el procedimiento seguido desde la recepción de los proyectos hasta la comunicación de los resultados, los criterios de evaluación y selección utilizados, responsables de determinar esos criterios, la participación o no de expertos externos dentro de las Comisiones de valoración, las características del seguimiento de los proyectos seleccionados, y la difusión de los resultados.

\section{Clarificación conceptual: "investigación educativa" y campos afines}

Para centrar el objeto de este estudio y, más en concreto, para el análisis de las convocatorias de las distintas instituciones, uno de los factores principales era determinar qué entendemos por "investigación educativa". Algo que en principio puede parecer fuera de discusión, no lo es en absoluto en el marco de las convocatorias, pues en ellas se mezclan acciones sobre la formación, el intercambio, la movilidad, la difusión, la creación de redes, la innovación y — dentro y/o fuera de cada acción — la investigación. Para resolver este problema de delimitación conceptual, adoptamos una definición que hemos utilizado como criterio de selección de convocatorias. Esta conceptualización incluye elementos convencionales sobre el concepto, recogidos en otras definiciones:

"La investigación educativa representa una actividad orientada hacia el desarrollo de un cuerpo organizado de conocimientos acerca de los acontecimientos que interesan a los edu- 
cadores. En este sentido, la investigación es un actividad orientada a la descripción, comprensión, explicación y/o transformación de la realidad educativa a través de un plan de indagación y análisis sistemático, crítico y público".

En cualquier caso, como señalaba el Plan de Investigación Educativa y de Formación del Profesorado (MEC, 1989, pp. 14 y ss.) "el concepto de investigación educativa no tiene unos límites netos y bien definidos, especialmente en el caso de la investigación aplicada a la innovación y la toma de decisiones para la planificación y política de la educación", como es el caso de los proyectos financiados generalmente por la Comisión Europea. En todo caso, como se señala en la misma obra, no toda propuesta de innovación, ni todo estudio descriptivo-estadístico debe considerarse una investigación pues no basta con modificar o describir los procesos y estructuras educativas, es necesario hacer esfuerzos de comprensión y/o explicación de los mismos. Estas son las pautas consideradas al incluir o excluir determinadas acciones comunitarias como objeto de este estudio.

En consecuencia, acordamos incluir también como objeto de este estudio —en algunos casos muy concretos - aquellos proyectos de "innovación educativa" que impliquen algún tipo de estudio o análisis en profundidad, pese a no tener el mismo alcance de los proyectos de investigación propiamente dicha. En todo caso, siendo este estudio un encargo del CIDE para la mejora de los criterios de política científica en educación, y atendiendo a la trayectoria de sus convocatorias, pensamos que no debían excluirse del estudio los programas europeos para la financiación de la innovación en educación.

Por otra parte, delimitar el campo de estudio supuso también adoptar decisiones acerca del objeto de investigación o de innovación, es decir la "educación" como parte de la definición del dominio a estudiar. Esta cuestión se plantea porque los Programas de la Comisión Europea no siempre se refieren a educación, sino a nuevas tecnologías, cohesión social, etc. Temas genéricos que no siempre encierran una perspectiva educativa que permita a los investigadores en este campo presentar proyectos de investigación, y a nosotras incluir dichos Programas como objeto de este estudio.

\section{Programas y acciones de la Comisión Europea: política científica}

Los Programas de la Comisión Europea cuyos procedimientos y criterios de evaluación/selección y seguimiento han sido analizados, son:

- Programa Sócrates

- Programa Leonardo da Vinci

- Programa Cultura 2000

- Programa Media Formación

- Programa Alfa

- Programa Equal

- Programa Eumedis

- Programa Connect

- Programa e-learning

- Programa Daphne 
- V Programa Marco

- VI Programa Marco

\section{Planificación de la recogida de información y documentación}

Una vez seleccionados los Programas Europeos que financian investigación e innovación educativa, se siguió un proceso de búsqueda de información y documentación relevante. En concreto, se definieron las vías por las que era necesario documentarse.

a) Sede de la Comisión Europea en Bruselas.

b) Web de la Comisión Europea y afines.

c) Agencias Nacionales.

Cuando fue preciso aclarar dudas y recabar información específica que no estaba difundida por ningún medio (impreso o telemático), se procedió a realizar entrevistas con una serie de responsables de instituciones implicadas en las convocatorias objeto de estudio. En concreto, se mantuvieron entrevistas (personales y/o telefónicas) con las siguientes instituciones:

- Responsable de Programas Europeos. Rectorado de la Universidad Complutense de Madrid.

- Vicerrectorado de Investigación de la Universidad Complutense de Madrid

- Vicerrectorado de Investigación (responsable de Programas Europeos) y Vicerrectorado de Relaciones Internacionales de la UNED.

- Agencia Nacional Sócrates.

- Entrevista con el responsable de la Comisión Europea de la Dirección General de Empleo y Asuntos Sociales y Red EURES.

- Responsables de la selección de proyectos del V Programa Marco (Madrid).

- Responsable del Programa Cultura 2000.

- Agencia Nacional del Programa Leonardo da Vinci.

- Responsable del Programa Equal en el Ministerio de Trabajo y Asuntos Sociales.

- Responsable de los Programas Marco de la Secretaría General del Plan Nacional de $\mathrm{I}+\mathrm{D}$.

- Responsables de los Programas E-Learning, Daphne y Connect en la Comisión Europea (Bruselas).

Hemos de señalar que a lo largo de las búsquedas de información y entrevistas realizadas se han encontrado documentos públicos relativos al tema objeto de estudio, a los procedimientos de presentación de proyectos, etc. En cambio, nos ha sorprendido la falta de transparencia y/o de publicidad dada a los criterios específicos que utilizan las Comisiones de evaluación (nacionales y europeas) para valorar y seleccionar los proyectos presentados a cada convocatoria.

En la documentación oficial consultada, los epígrafes relativos a criterios de evaluación se refieren primordialmente a los criterios formales de presentación de proyectos, es decir, apartados que debe incluir, datos imprescindibles, extensión del proyecto, requisitos de 
Análisis de los procedimientos y criterios...

idioma, pasos a seguir desde la presentación del proyecto hasta la superación de las fases de valoración, plazos de entrega, etc. Sin embargo, no se mencionaban los criterios utilizados para seleccionar/financiar unos proyectos en detrimento de otros. Nuestro propósito de localización de dichos criterios se vio dificultada por varios motivos:

1) Insuficiente difusión de los criterios específicos de evaluación de proyectos.

2) Falta de claridad al no señalar explícitamente qué acciones financian investigación educativa y cuáles no.

3) Burocratización del proceso de presentación, valoración y seguimiento de proyectos, dado que especialmente los programas de acciones descentralizadas tienen una fase de valoración en las Agencias Nacionales de cada país y una fase posterior de valoración en otros países miembros de la UE por un comité de evaluación.

4) Los responsables de los organismos consultados (de las Agencias Nacionales especialmente), no ofrecen información precisa sobre el tema (por desconocimiento, no por desinterés en la mayoría de los casos).

5) Escasa bibliografía actualizada sobre el tema.

6) No todos los documentos fundamentales relativos a los Programas Europeos tienen una versión en español, lo que permitiría sacar muchas conclusiones (el español es una lengua comunitaria, y en ella debe facilitarse información a los investigadores españoles).

Las dificultades relativas a la falta de difusión y precisión de los criterios de evaluación/selección, se ha compensado por una ardua búsqueda de fuentes complementarias que nos aportan la información ausente en los documentos publicados, como las entrevistas con los responsables de los programas y la solicitud de documentos internos.

Sin embargo, hay que decir que en el V y VI Programa Marco existe una difusión clara y detallada de los criterios generales que rigen estos Programas y de los criterios específicos que se aplican a cada Acción, ofreciendo incluso una sencilla clasificación. Además, se especifica también el sistema de ponderación que se asigna a dichos criterios, permitiendo así su priorización. Por ello, decidimos centrarnos en el V Programa Marco y en el VI Programa Marco y asumir parcialmente dicha clasificación de criterios para nuestro estudio, como ya se ha señalado.

\section{Metodología de trabajo: clasificación, comparación y análisis de la información}

Una vez seleccionados los Programas Europeos que financian investigación e innovación educativa, y localizada la información relevante, se siguió un proceso de análisis inductivo-deductivo, acometiéndose primero el estudio detallado de cada Programa y de sus correspondientes acciones en función de los conceptos básicos (investigación educativa) para, después, sintetizar los procedimientos y criterios de evaluación/selección predominantes. Por ello se ha realizado:

2) Un estudio analítico y cualitativo, porque se ha analizado el contenido de cada programa para:

a) identificar los programas y las acciones europeas que financian proyectos de investigación educativa, y 
b) el procedimiento y criterios de evaluación y seguimiento de estos programas.

De esta forma, se ha obtenido una síntesis de criterios de acuerdo a una clasificación que posteriormente explicaremos.

2) Un estudio comparativo porque, tras desmenuzar los criterios de cada programa, se ha realizado un recuento de los criterios similares - de acuerdo a categorías definidas previamente - y se ha obtenido una síntesis de los criterios más frecuentes en función de si la Acción es centralizada o descentralizada, el contenido o ámbito de aplicación del Programa, los destinatarios, los posibles países participantes, etc.

3) Un estudio transversal, no longitudinal, dado que se han extraído los procedimientos y criterios de cada programa en la fase actual en la que se encuentran, no los criterios que ha utilizado cada programa a lo largo de períodos anteriores.

Por tanto, en este proceso se han buscado:

1) El procedimiento y los criterios generales de evaluación/selección de proyectos que se aplican en cada Programa Europeo.

2) Los criterios específicos de cada Acción, y

3) Los criterios de la última Convocatoria anual de cada programa.

\section{Análisis de documentación disponible: categorías de análisis}

Para abordar la ingente documentación encontrada en cada Programa Europeo, tuvimos que adoptar un sistema de trabajo que permitiese el análisis pormenorizado de cada Programa y la identificación relevante para este estudio. Para ello, seguimos el siguiente sistema de trabajo:

a) Análisis de los programas europeos: se ha realizado una tabla descriptiva por cada Programa y por cada acción. En ella, se señala si es una acción centralizada o descentralizada, los objetivos que tiene y las acciones y actividades que pueden financiar proyectos de investigación y/o innovación educativa.

b) Procedimientos y Criterios de evaluación y Seguimiento de Proyectos de INVESTIGACIÓN EDUCATIVA: por cada Programa Europeo se ha sintetizado tres tipos de información:

1) Procedimientos utilizados PaRA la EVAluación/SELECCiÓN DE PROYECTOS DE INVESTIGACIÓN EDUCATIVA: Dada la variedad y multiplicidad de procedimientos, es importante analizar el proceso que debe seguir cada proyecto para ser evaluado, las fases de valoración, quién evalúa o compone las Comisiones de evaluación y la frecuencia de selección de proyectos. En este punto, es importante señalar que el procedimiento suele variar significativamente dependiendo de si la acción es centralizada (es decir, los proyectos se evalúan en Bruselas), descentralizada (los proyectos se evalúan en las Agencias Nacionales) o mixta (consta de dos fases, en la Agencia Nacional y en Bruselas), dado que los criterios de evaluación pueden variar en función de la Agencia Nacional, debido a las prioridades temáticas de ese país o a las comisiones de expertos que lo valoren.

2) Criterios utilizados Para la evaluación/selección de Proyectos DE INVEstiGACIÓN EDUCATIVA: al analizar los programas se observó que se utilizaban crite- 
rios muy heterogéneos, que no estaban priorizados, que muy pocos tenían asignada algún tipo de ponderación y que, no siempre, los criterios respondían a una fácil clasificación que permitiera su comparación. Por ello, establecimos previamente unas categorías para poder clasificar los criterios, facilitar la síntesis posterior y la obtención de conclusiones de utilidad. Para definirla nos basamos en la clasificación utilizada por el V Programa Marco y añadimos una nueva categoría de clasificación - "criterios referidos al tema de investigación"- porque aportaba claridad a la clasificación de criterios. Así pues, las categorías de análisis utilizadas fueron las siguientes:

Criterios temáticos: en esta categoría hemos incluido los criterios que priorizan determinados temas, campos o contenidos de investigación. Este tipo de criterios suelen incluirse en las Convocatorias anuales de cada país que se valoran en las Agencias Nacionales (descentralizadas).

Calidad e innovación científica y tecnológica: en ella se localizan los criterios relativos a la calidad de la investigación propuesta (en términos de calidad técnica del proyecto: objetivos bien definidos, metodología adecuada, etc.) y su contribución al logro de los objetivos del Programa europeo. También se incluyen aquí criterios de valoración del grado de innovación.

Valor añadido comunitario y contribución a las políticas de la UE: aquí se han ubicado los criterios relativos al impacto europeo del proyecto, al valor añadido que puede generar el proyecto por la contribución de diferentes países, y la contribución al desarrollo de las políticas definidas por la Unión Europea.

Contribución a los objetivos sociales comunitarios: en esta categoría hemos situado los criterios referidos al logro de objetivos sociales importantes para la UE, como la calidad de vida, la igualdad de hombres y mujeres, la salud, la seguridad, el empleo, el medio ambiente, etc.

Desarrollo económico y prospectiva científica y tecnológica: se trata de criterios de evaluación del crecimiento, desarrollo económico e impacto estratégico del proyecto y su potencial para mejorar la competitividad. Se incluyen también las valoraciones de las estrategias de difusión de los resultados del proyecto.

Recursos, asociación y gestión: esta categoría se refiere a los criterios que valoran la calidad de los recursos y la gestión del proyecto, así como a los miembros que forman la asociación y su grado de implicación.

Otros: en esta categoría se incorporan los criterios - pocos- que no han podido clasificarse en las categorías anteriores.

3) Seguimiento de los proyectos de inVestigación educativa: se ha analizado el proceso de seguimiento que se utiliza en cada programa para valorar el progreso de los proyectos financiados. Por ello, se indica si en ese programa se efectúa algún tipo de seguimiento o no, y en su caso, qué documentación se solicita, en qué momento se pide (durante la realización del proyecto o sólo al final) y las medidas que se toman en caso de que los investigadores incumplan alguno de los compromisos (no entregar la memoria, no cumplir los plazos, etc.). 


\section{Aportaciones del estudio sobre los Programas Europeos que financian investigación educativa}

\section{Sobre los Programas Generales}

Tras realizar el análisis de Programas Sócrates, Leonardo da Vinci, Cultura 2000, Media Plus, Alpha, Equal, Eumedis, Connect, e-learning y Daphne, este estudio aporta la información siguiente:

1) Los procedimientos de evaluación utilizados para presentar los proyectos.

2) Una síntesis de los criterios generales de evaluación/selección, que delimitan los parámetros en los que se deben mover los proyectos de investigación presentados a cada Programa. Estos criterios generales son comunes a cada una de las acciones que incluye el Programa.

3) Unos criterios específicos de valoración para cada una de las Acciones.

4) El seguimiento que se da a los proyectos financiados.

\section{Sobre el V y VI Programa Marco de Investigación}

Las aportaciones principales de este estudio en relación al V y VI Programa Marco, ha sido la obtención de:

1) Síntesis del procedimiento general de evaluación de los proyectos de investigación educativa (a partir del documento marco de la Comisión).

2) Información sobre los criterios generales y comunes de evaluación/selección, clasificados en las distintas Acciones de estos dos Programas Marco.

3) Información sobre los criterios específicos de valoración de cada una de las Acciones.

4) Síntesis del seguimiento que se da a los proyectos financiados.

En líneas generales, podemos decir que constituyen una herramienta útil tanto para los investigadores en materia educativa, como para las instituciones responsables de planificar, evaluar y financiar investigación educativa, como el propio CIDE o sus posibles homólogos en las Comunidades Autónomas. Algunas de las razones de su utilidad son las siguientes:

- Utilidad para los investigadores:

1) Se ofrece una síntesis de todos los Programas y acciones europeas que financian proyectos de investigación educativa, de forma que todas las instituciones o grupos de investigación interesados puedan acceder fácilmente a la oferta existente (en las Acciones concretas que financian investigación educativa).

2) Se muestra una síntesis de las prioridades temáticas de la Guía del Candidato de cada Programa, para ser tenidas en cuenta a la hora de orientar los proyectos de investigación.

3) Se facilita una Guía de los criterios generales de evaluación/selección de cada Programa, con los criterios específicos que definen cada Acción. Esto permite a 
los investigadores ubicar y perfilar mejor sus proyectos de investigación, y aumentar las probabilidades de selección.

4) Se proporciona un listado de los Criterios utilizados para valorar y seleccionar los proyectos que se van a financiar en cada Acción. Dichos criterios están clasificados en función de la temática y dimensión europea, de los criterios regionales, de los destinatarios, de la metodología y del equipo participante.

5) Constituye en conjunto una herramienta útil para estructurar y afinar con mayor rigor científico cualquier proyecto de investigación educativa.

- Utilidad para las instituciones responsables de planificar, financiar y evaluar la investigación educativa:

1) Se ofrece una panorámica del estado de la cuestión del proceso de selección/financiación de proyectos de investigación educativa en la UE.

2) Se proporciona una síntesis de los Criterios de evaluación de proyectos más utilizados en la Comisión Europea, identificados en función de la frecuencia de aplicación y del tema objeto de cada Programa. Esto da una idea del peso e importancia que tiene cada criterio y, en definitiva, de la orientación y recomendaciones que están guiando las políticas sobre investigación educativa de la Comisión.

3) El Informe constituye un documento útil para reflexionar sobre los "aciertos" y "errores" de la organización y puesta en práctica de Programas y Acciones que financian investigación educativa a escala Europea. Este proceso podrá ayudar a mejorar la planificación científica y técnica de las instituciones nacionales y autonómicas competentes en materia de investigación educativa.

\section{Resultados y conclusiones generales del estudio}

Este estudio, realizado para el CIDE, constituye la primera fase de un trabajo más amplio de ámbito estatal, cuyo objetivo último es la elaboración de una Guía para la evaluación y seguimiento de proyectos de investigación educativa.

Esta iniciativa se basa en la convicción sobre la importancia de establecer una política adecuada para la asignación de los recursos destinados a la investigación, en este caso concreto, en materia educativa. Los procedimientos y criterios de evaluación, selección y seguimiento de los proyectos de investigación funcionan de hecho como indicadores de lo que desde los organismos públicos se considera una práctica investigadora de calidad. Por esta razón tienen un papel muy importante en el incremento de unos tipos y modelos de investigación y en la extinción progresiva de otros.

Como se afirma en un informe de la O.C.D.E. (1995, pp. 3 y 13) sobre las tendencias y retos de la investigación educativa ligada al desarrollo (I + D), "la necesidad de mejorar su relevancia y eficiencia pasa por el alineamiento y la vinculación de los diversos intereses de tres "comunidades": investigadores, políticos y prácticos de la educación... Al mismo tiempo, es importante no implantar una política científica de la inmediatez, si deseamos que los equipos de investigación acumulen habilidades y conocimientos y se creen y mantengan las redes que constituyen el factor en la difusión y utilización de los logros de la investigación educativa". 
Hoy es mucha la evidencia teórica y empírica disponible para justificar la necesidad de disponer de un procedimiento contrastado y público, que permita a los organismos financiadores conseguir sus objetivos, a saber:

1) El fomento de la cantidad y calidad de actividad investigadora.

2) La vinculación de la investigación con el desarrollo — con la mejora de la calidad de la educación, y de nuestro conocimiento básico acerca de los procesos de enseñanza, de aprendizaje y del sistema educativo en general-, donde los protagonistas de la intervención educativa tengan un papel activo como investigadores.

3) La rentabilidad de los recursos públicos destinados a investigación —objetivo muy relacionado con el anterior.

4) La formación de los distintos participantes en la investigación -investigadores e "investigados".

Pese a las dificultades encontradas para localizar información fidedigna sobre los procedimientos y criterios de evaluación empleados en distintos organismos europeos (Comisión Europea, NFER e INRP) para la evaluación de la investigación educativa, creemos que este estudio muestra tendencias de importancia que resumiremos a continuación.

Después de analizar, comparar y valorar la información recogida en distintos organismos nacionales y multilaterales, los resultados y conclusiones obtenidos se organizan en torno a los tres ejes fundamentales del estudio:

1) Procedimientos de evaluación.

2) Criterios de evaluación/selección de proyectos para su financiación.

3) Seguimiento de los proyectos subvencionados.

A continuación se presenta, para cada uno de los ejes mencionados, la comparación de las prácticas de cada uno de los organismos, así como las conclusiones generales.

\section{Procedimientos de evaluación}

En este estudio se ha entendido por procedimiento de evaluación, la respuesta a la pregunta ¿quién evalúa los proyectos de investigación educativa presentados a las distintas convocatorias? (Comisiones internas/externas de expertos estables/temporales; perfil de los evaluadores: investigadores, funcionarios, Comisiones mixtas, etc.).

Sabiendo de antemano la distinta naturaleza, fines y estructura de cada uno de estos organismos, no es extraño observar cómo, en el caso de la NFER y el INRP los evaluadores sean generalmente miembros de la propia institución (Tabla 1).

Reconociendo que la información que hemos podido obtener sobre los procedimientos de evaluación de los tres organismos estudiados no es muy completa - lo que ya da una primera idea de lo restrictivo de la información pública acerca de sus prácticas evaluativas, siendo un aspecto que los distancia enormemente de lo que es habitual en el caso del CIDE español-, a partir de los datos de que disponemos podemos aventurar que en el caso de los organismos nacionales no se da una evaluación por Comisiones de expertos variables, independientes y ajenas a la institución, a diferencia de la Comisión Europea. 
TABLA 1: Procedimiento de evaluación de proyectos en los organismos europeos objeto de estudio

\begin{tabular}{|c|c|c|c|c|c|}
\hline \multirow{2}{*}{\multicolumn{2}{|c|}{$\begin{array}{c}\text { O. MULTILATERAL } \\
\text { Comisión Europea }\end{array}$}} & \multicolumn{4}{|c|}{ ORGANISMOS NACIONALES } \\
\hline & & \multicolumn{2}{|c|}{ NFER (R. Unido) } & \multicolumn{2}{|c|}{ INRP (Francia) } \\
\hline $\begin{array}{l}\text { Acciones } \\
\text { centralizadas }\end{array}$ & $\begin{array}{l}\text { Acciones } \\
\text { descentralizadas }\end{array}$ & $\begin{array}{l}\text { Dirección de } \\
\text { proyectos } \\
\text { con } \\
\text { financiación } \\
\text { externa. }\end{array}$ & $\begin{array}{l}\text { Proyectos } \\
\text { propios }\end{array}$ & $\begin{array}{l}\text { Dirección de } \\
\text { proyectos } \\
\text { con } \\
\text { financiación } \\
\text { externa. }\end{array}$ & $\begin{array}{l}\text { Proyectos } \\
\text { propios }\end{array}$ \\
\hline $\begin{array}{l}\text { Evalúa la } \\
\text { correspondiente } \\
\text { unidad de la } \\
\text { dirección general de } \\
\text { la Comisión en } \\
\text { Bruselas, con la } \\
\text { colaboración de } \\
\text { expertos externos. } \\
\text { Puede haber } \\
\text { procedimientos de } \\
\text { selección de } 1 \text { fase } \\
\text { (selección directa) o } \\
\text { de } 2 \text { fases (con lista } \\
\text { corta). Puede haber } \\
\text { algunas variaciones } \\
\text { mínimas por } \\
\text { programas. }\end{array}$ & $\begin{array}{l}\text { Procedimiento en } \\
\text { dos fases: 1) } \\
\text { Evaluación por la } \\
\text { Agencia Nacional de } \\
\text { Pr. Europeos; 2) La } \\
\text { Comisión consulta a } \\
\text { las Agencias } \\
\text { Nacionales de los } \\
\text { países implicados en } \\
\text { el Proyecto.Puede } \\
\text { haber algunas } \\
\text { variaciones por } \\
\text { programas, que } \\
\text { suelen consistir en } \\
\text { recabar la } \\
\text { colaboración de } \\
\text { expertos externos. }\end{array}$ & \multicolumn{2}{|c|}{$\begin{array}{l}\text { En ambos casos evalúa } \\
\text { el Departamento } \\
\text { responsable de cada } \\
\text { área de proyectos (no } \\
\text { hay evaluación externa). }\end{array}$} & \multicolumn{2}{|c|}{$\begin{array}{l}\text { En ambos casos, } \\
\text { evalúan las Unidades de } \\
\text { Investigación del } \\
\text { Instituto (formadas por } \\
\text { investigadores } \\
\text { permanentes y } \\
\text { profesores asociados). }\end{array}$} \\
\hline
\end{tabular}

\section{Criterios de evaluación y selección de proyectos para su financiación}

Con relación a este eje habría que diferenciar entre, criterios generales de evaluación, y criterios específicos por programas. Sobre estos últimos, sólo tenemos información completa de la Comisión Europea que, en todo caso, nos permite obtener conclusiones de alcance general.

\section{Criterios generales}

Una primera conclusión en este aspecto es que, a partir de la información disponible, es difícil comparar los criterios generales utilizados por cada uno de los tres organismos estudiados, en primer lugar porque el grado de generalidad de los de la Comisión Europea es mayor (los de los otros dos organismos responden más a lo que son criterios específicos de los programas y acciones europeas), y en segundo lugar porque - como criterios generales que son - se ajustan a los fines de cada una de las instituciones, que son bastante distintos entre sí. Sin embargo, es interesante considerarlos conjuntamente (Tabla 2). 


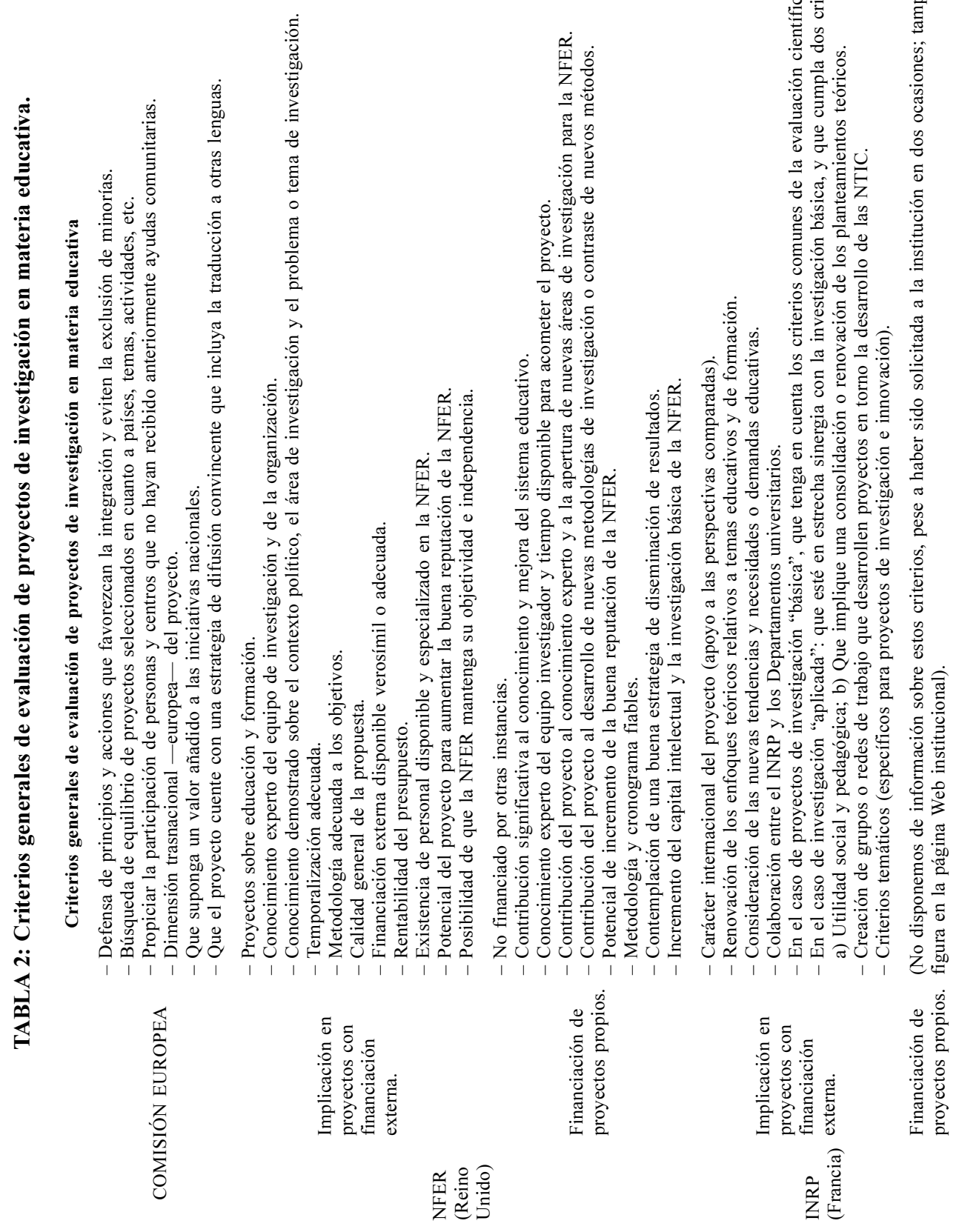


Como anunciábamos al principio de este epígrafe, tan sólo hay coincidencia en un criterio entre la C.E. y la NFER: "la contemplación en el proyecto de una buena estrategia de difusión o diseminación de resultados", criterio que tiene a nuestro juicio una gran importancia por lo que supone de garantía de visibilidad — hacia la sociedad y la comunidad científica y profesional— de las aportaciones de una investigación.

Ahora bien, con carácter general, de este tipo de criterios se puede extraer una conclusión de interés para el establecimiento de una guía de procedimiento y unos criterios generales (estatales) de evaluación de proyectos de investigación educativa, a iniciativa del CIDE, con la colaboración de las CC.AA.

Mientras que la NFER y la INRP emplean los criterios habituales en cualquier convocatoria de esta naturaleza (experiencia del equipo investigador, adecuación metodológica, etc.), a excepción de algunos criterios más originales - como la "contribución del proyecto a la reputación de la institución", o "que la institución mantenga su objetividad e independencia" utilizados por la NFER-, son algunos de los criterios generales utilizados por la Comisión Europea los que nos plantean algunas líneas prospectivas de más interés desde la perspectiva de este estudio. Nos permiten hacer una trasposición desde el ámbito europeo, al del Estado español de las Autonomías, de forma que el organismo al que la Constitución reserva la competencia en materia de fomento y mejora de la calidad de la investigación educativa (el CIDE), pudiera mantener convocatorias de carácter estatal, cuyos criterios de evaluación/selección de proyectos fueran:

1) Defensa de principios y acciones que favorezcan la integración y eviten la exclusión de minorías (étnicas, culturales, lingüísticas, etc. de todo tipo).

2) Búsqueda de equilibrio de proyectos seleccionados en cuanto a comunidades Autónomas, temas, actividades, etc.

3) Propiciar la participación de personas y centros que no hayan recibido anteriormente ayudas a la investigación.

4) Dimensión trasregional —nacional— del proyecto.

5) Que suponga un valor añadido a las iniciativas en materia de investigación educativa de la Comunidades Autónomas.

6) Que el proyecto cuente con una estrategia de difusión convincente que incluya la traducción a otras lenguas del Estado (catalán, eusquera, gallego, castellano, etc.).

7) Creación de una Red de Información Estatal sobre investigación educativa, semejante a la Red Eurydice, pero con acceso no sólo institucional, sino también para los investigadores.

\section{Criterios específicos}

En la información disponible sobre la NFER y el INRP apenas se reflejan criterios específicos, por lo que analizaremos los establecidos por la Comisión Europea para sus diferentes programas y acciones.

$\mathrm{Al}$ analizar los Programas y acciones europeas hemos observado que se utilizan criterios de evaluación muy heterogéneos, que no están priorizados, que muy pocos tienen asignada algún tipo de ponderación, y que no siempre los criterios responden a una fácil clasificación 
que permitiera su posterior comparación. Por ello, hemos establecido previamente unas categorías para clasificar los criterios de los distintos programas y acciones, y facilitar así la síntesis y la obtención de conclusiones de utilidad para el CIDE.

Para definir estas categorías nos hemos basado en la clasificación utilizada por el V Programa Marco, aunque hemos añadido una nueva categoría de clasificación - "criterios referidos al tema de investigación"- - ya que aportaba claridad a la clasificación. La clasificación de criterios de evaluación obtenida se muestra en la tabla 3.

TABLA 3: Categorías de criterios de evaluación. Programas y acciones europeas.

\begin{tabular}{|l|l|}
\hline \multicolumn{2}{|c|}{ Categorías de criterios de evaluación. Programas y acciones europeas } \\
\hline Criterios temáticos & $\begin{array}{l}\text { En esta categoría hemos incluido los criterios que priorizan } \\
\text { determinados temas, campos o contenidos de investigación. Este tipo de } \\
\text { criterios suelen incluirse en las Convocatorias anuales de cada país que } \\
\text { se valoran en las Agencias Nacionales (en las acciones descentralizadas). }\end{array}$ \\
\hline $\begin{array}{l}\text { Calidad e innovación } \\
\text { científica y } \\
\text { tecnológica }\end{array}$ & $\begin{array}{l}\text { En ella se localizan los criterios relativos a la calidad de la investigación } \\
\text { propuesta (en términos de calidad técnica del proyecto: objetivos bien } \\
\text { definidos, metodología adecuada, etc.) y su contribución al logro de los } \\
\text { objetivos del Programa europeo. También se incluyen aquí criterios de } \\
\text { valoración del grado de innovación. }\end{array}$ \\
\hline $\begin{array}{l}\text { Valor añadido } \\
\text { comunitario y } \\
\text { contribución a las } \\
\text { políticas de la UE }\end{array}$ & $\begin{array}{l}\text { Aquí se han ubicado los criterios relativos al impacto europeo del } \\
\text { proyecto, al valor añadido que puede generar el proyecto por la } \\
\text { contribución de diferentes países, y la contribución al desarrollo de las } \\
\text { políticas definidas por la Unión Europea. }\end{array}$ \\
\hline $\begin{array}{l}\text { Contribución a los } \\
\text { objetivos sociales } \\
\text { comunitarios }\end{array}$ & $\begin{array}{l}\text { En esta categoría hemos situado los criterios referidos al logro de } \\
\text { objetivos sociales importantes para la UE, como la calidad de vida, la } \\
\text { igualdad de hombres y mujeres, la salud, la inserción social, la } \\
\text { seguridad, el empleo, el medio ambiente, etc. }\end{array}$ \\
\hline $\begin{array}{l}\text { Desarrollo } \\
\text { económico, y } \\
\text { prospectiva científica } \\
\text { y tecnológica }\end{array}$ & $\begin{array}{l}\text { Se trata de criterios de evaluación del crecimiento, desarrollo económico } \\
\text { e impacto estratégico del proyecto y su potencial para mejorar la } \\
\text { competitividad. Se incluyen también las valoraciones de las estrategias } \\
\text { de difusión de los resultados del proyecto. }\end{array}$ \\
\hline $\begin{array}{l}\text { Recursos, asociación } \\
\text { y gestión }\end{array}$ & $\begin{array}{l}\text { Esta categoría se refiere a los criterios que valoran la calidad de los } \\
\text { recursos y de la gestión del proyecto, así como a los miembros que } \\
\text { forman la asociación y su grado de implicación. }\end{array}$ \\
\hline Otros & $\begin{array}{l}\text { En esta categoría se incorporan los criterios -pocos- que no han podido } \\
\text { clasificarse en las categorías anteriores. }\end{array}$ \\
\hline
\end{tabular}

Dada la limitación de espacio para este artículo $^{4}$, nos vamos a limitar a continuación a sintetizar las conclusiones principales que es posible extraer de su análisis.

4 Las personas interesadas en consultar todos los criterios de evaluación/selección de cada Programa o acción comunitaria, pueden ponerse en contacto con las autoras de este artículo. 


\section{Criterios temáticos}

La propia estructura de los Programas Europeos que financian investigación educativa, responde a un criterio temático (por ejemplo, el Programa Sócrates responde a un tema general de investigación: la educación — cooperación europea para mejorar su calidad-, y tiene distintas acciones - Comenius, Grundvig, etc. - que también contemplan proyectos sobre temas específicos en educación; igual ocurre con el Programa Leonardo, cuyo tema central es la formación orientada al empleo, etc.).

¿Qué puede significar esto para un Instituto de Investigación como el CIDE, o incluso para organismos similares de carácter autonómico? La idea sería crear Programas amplios de investigación sobre temas básicos y estables (como educación, formación y empleo, nuevas tecnologías, etc.), que se subdividieran por acciones específicas atendiendo a criterios temáticos. Esto permitiría mantener siempre abiertas líneas de investigación estables y generales y, dentro de cada una de ellas, líneas de investigación más concretas que podrían variar por períodos razonables de tiempo (bienio o quinquenio), en función de prioridades y demandas sociopolíticas.

\section{El criterio de "calidad e innovación científica y tecnológica" del proyecto}

Se trata de una categoría clásica, que incluye criterios de calidad científico-técnica del proyecto (objetivos bien definidos, metodología adecuada, gestión, cronograma, viabilidad, etc.), así como criterios de valoración del grado de innovación de los métodos de investigación y los recursos tecnológicos empleados. En términos generales, casi todos los organismos suelen mostrarse coincidentes en el empleo y la naturaleza de este tipo de criterios.

Tan sólo hay un aspecto que se incorpora de manera irregular, y es la relación entre la teoría y la práctica, la contemplación de las sinergias entre la investigación básica y la aplicada. De hecho, tan sólo el INRP contempla este tipo de criterios generales ("Renovación de los enfoques teóricos relativos a temas educativos y de formación", "En el caso de proyectos de investigación"básica", que tenga en cuenta los criterios comunes de la evaluación científica", "En el caso de investigación "aplicada": que esté en estrecha sinergia con la investigación básica, y que cumpla dos criterios:

a) Utilidad social y pedagógica;

b) Que implique una consolidación o renovación de los planteamientos teóricos").

La inclusión de este tipo de criterios en las convocatorias públicas no hace sino incorporar los criterios epistemológicos actuales sobre la investigación que puede considerarse científica, y no una mera generalización inductiva difícil de interpretar y de integrar en el ciclo del conocimiento.

\section{El criterio del "Valor añadido comunitario y contribución a las políticas de la UE"}

Este es un criterio muy válido, tanto para comunidades de países, como de estados, regiones o comunidades autónomas, como es el caso español. Como ya señalábamos con relación a los criterios generales de evaluación, también puede ser un criterio específico para 
TABLA 5: Seguimiento de los proyectos subvencionados.

\begin{tabular}{|c|c|c|}
\hline Comisión Europea & NFER & INRP \\
\hline $\begin{array}{l}\text { - Existe un "Contrato } \\
\text { institucional". } \\
\text { - Se solicitan informes } \\
\text { parciales y final. } \\
\text { - Los beneficiarios deben } \\
\text { estar dispuestos a responder } \\
\text { a las preguntas de } \\
\text { evaluadores y auditores, y a } \\
\text { cumplimentar encuestas de } \\
\text { evaluación y supervisión. } \\
\text { - Los pagos parciales y final } \\
\text { se efectúan a tarea realizada } \\
\text { y demostrada, de acuerdo al } \\
\text { contrato. } \\
\text { - Las medidas sancionadoras } \\
\text { no se especifican con } \\
\text { carácter general, sino en } \\
\text { cada contrato-programa } \\
\text { (suele suponer la ausencia } \\
\text { de pago del segmento } \\
\text { correspondiente e, incluso, e } \\
\text { reembolso de las cantidades } \\
\text { recibidas). }\end{array}$ & $\begin{array}{l}\text { - El seguimiento lo hace cada } \\
\text { Director de Proyecto (en } \\
\text { caso de proyectos internos) } \\
\text { y por un Comité de } \\
\text { Dirección (en caso de } \\
\text { proyectos externos) de la } \\
\text { NFER, a través de una base } \\
\text { de datos informatizada. } \\
\text { - Se piden informes parciales } \\
\text { y final. } \\
\text { - Se hace un seguimiento } \\
\text { mensual de la ejecución del } \\
\text { presupuesto de cada } \\
\text { proyecto. } \\
\text { - En caso de incumplimiento } \\
\text { son varias las medidas: } \\
\text { conceder más apoyo y } \\
\text { ayudas directas de la NFER } \\
\text { a la investigación y/o a la } \\
\text { redacción del Informe; } \\
\text { cambiar miembros del } \\
\text { equipo o incorporar nuevos } \\
\text { expertos; reconducir el plan } \\
\text { de trabajo; solicitar } \\
\text { financiación adicional; } \\
\text { prorrogar el plazo de } \\
\text { finalización. }\end{array}$ & $\begin{array}{l}\text { - La supervisión la realizan } \\
\text { los Departamentos o } \\
\text { Unidades de Investigación } \\
\text { del propio Instituto. } \\
\text { - Deben entregarse informes } \\
\text { parciales y final. } \\
\text { - (No se dispone de más } \\
\text { información al respecto). }\end{array}$ \\
\hline
\end{tabular}

la promoción y financiación de líneas de investigación sobre temas o iniciativas concretas. Indudablemente, una política científica en materia educativa, es decir, convocatorias de investigación educativa que promuevan proyectos de investigación que abordan un tema en el ámbito del estado - y no sólo de la ciudad, la provincia o la C.A.— permitiría a los equipos de investigadores (organizados en redes universitarias u otras) acometer estudios comparados - sobre respuestas a la educación de los inmigrantes, modelos de orientación educativa, eficacia de políticas compensatorias, etc.- - De lo contrario, este tipo de estudios sólo podría ser acometidos por organismos públicos o privados. Asimismo, permitirían dar auténtico sentido al término "valor añadido", porque podrían completar, ampliar y enriquecer las iniciativas regionales.

\section{El criterio de la "Contribución a los objetivos sociales comunitarios"}

Con la presencia de criterios de evaluación referidos a proyectos de investigación educativa sobre el logro de objetivos sociales importantes y de ámbito nacional, como la calidad de la educación para todos, la igualdad de hombres y mujeres, la inserción social, la 
formación y orientación para el empleo, la igualdad de oportunidades para discapacitados, la conservación del medio ambiente, la cohesión social y económica entre regiones, la lucha contra la violencia, el racismo y la xenofobia, etc.

\section{El criterio del "Desarrollo económico y la prospectiva científica y tecnológica"}

Se trata de evaluar el posible impacto estratégico del proyecto, así como su potencial para abrir nuevas vías de investigación. Por ello, parece un criterio a considerar en cualquier convocatoria pública, regional o estatal.

\section{Recursos, asociación y gestión}

Un tipo de criterios que suelen incluirse en la mayoría de las convocatorias de proyectos de investigación educativa, es la experiencia y trayectoria del equipo de investigación, la adecuación de los recursos a utilizar para desarrollar el proyecto y la calidad del proceso de gestión del mismo. Sin embargo, no siempre está claro el sentido de la valoración: se puede primar la experiencia del equipo (como es el caso de la NFER y el INRP), o premiar los nuevos equipos o redes de investigadores (la C.E.); con frecuencia, la evaluación de la calidad de la gestión del proyecto varía mucho en el grado de exigencia (se acepta un simple cronograma del proyecto por fases, o se exige un cronograma detallado (horas o meses dedicados por cada investigador a cada tarea; disponibilidad temporal justificada, etc.). En las convocatorias de organismos multilaterales (C.E., BID, OEA, Banco Mundial, OCDE, etc.) suele exigirse la pormenorización de los procesos de gestión y realización del proyecto, así como de la ejecución del presupuesto.

Este tipo de criterios, todos ellos recogidos en las convocatorias europeas, no siempre se incluyen en las de los organismos nacionales (Tabla 4).

TABLA 4: Tipología de criterios utilizados por los distintos organismos estudiados.

\begin{tabular}{|l|c|c|c|}
\hline \multicolumn{1}{|c|}{ Tipos de criterios / organismo } & C.E. & NFER & INRP \\
\hline Criterios temáticos & $\mathrm{X}$ & $\mathrm{X}$ & $\mathrm{X}$ \\
\hline Calidad e innovación científica y tecnológica & $\mathrm{X}$ & $\mathrm{X}$ & $\mathrm{X}$ \\
\hline $\begin{array}{l}\text { Valor añadido comunitario y contribución a las políticas del } \\
\text { ámbito territorial de referencia }\end{array}$ & $\mathrm{X}$ & & $\mathrm{X}$ \\
\hline Contribución a los objetivos sociales comunes & $\mathrm{X}$ & & $\mathrm{X}$ \\
\hline Desarrollo económico, y prospectiva científica y tecnológica & $\mathrm{X}$ & $\mathrm{X}$ & $\mathrm{X}$ \\
\hline Recursos, asociación y gestión & $\mathrm{X}$ & $\mathrm{X}$ & $\mathrm{X}$ \\
\hline
\end{tabular}

En este caso se aprecia sobre todo, la inclusión o no de la perspectiva trasnacional (europea o internacional) entre los criterios de evaluación/selección de proyectos, cuya ausencia destaca en el caso de la NFER británica. 


\section{Seguimiento de los proyectos subvencionados}

Tras recoger la información disponible —algo vaga en general, salvo en el caso de los Programas Marco de la Comisión Europea-, es posible apreciar bastante homogeneidad en el enfoque del seguimiento de proyectos entre las tres instituciones estudiadas (entrega de informes, supervisión continua, pagos contra tarea realizada, etc.), aunque cierta heterogeneidad en cuanto al tipo de medidas sancionadoras en caso de incumplimiento de los objetivos o los plazos del proyecto (Tabla 5).

Como se puede observar, las prácticas de la NFER en caso de incumplimiento van más en la línea de entender y resolver los problemas de incumplimiento (insuficientes recursos financieros, falta de recursos humanos especializados, necesidad de renegociar el plazo de finalización e incluso los objetivos del proyecto, etc.), lo que lleva a pensar que la NFER dispone de medios y personal que puede ayudar a los equipos de investigación, con preferencia a las medidas sancionadoras más estrictas, como en el caso de la Comisión Europea.

En conjunto, puede decirse que la información disponible más útil para los propósitos de este estudio es la proporcionada por las convocatorias de la Comisión Europea por varias razones:

1) Porque la propia dificultad que tiene para el investigador "de a pie" acceder a los procedimientos y criterios de evaluación/selección y seguimiento de proyectos es toda una lección de lo que debe evitarse a toda costa. La propia opacidad y complejidad de las convocatorias — si no se dispone de la ayuda de un guía experimentado - , permite afirmar que esto está suponiendo de entrada una primera "criba" para investigadores/proyectos. Este tipo de situaciones deben evitarse en cualquier organismo que pretenda dar cabida a los profesionales de la educación (profesores, maestros, orientadores, educadores sociales, etc.) en la investigación educativa financiada con fondos públicos. Por otra parte, refuerzan el valor de las Guías prácticas para investigadores en busca de financiación para sus proyectos, así como el de la celebración de reuniones o sesiones informativas y de creación de redes temáticas, promovidas por los organismos financiadores.

2) Incorporan una estructura temática en sus programas, replicable en organismos nacionales o regionales, que incentivan líneas de investigación permanentes y temporales.

3) Porque manteniendo un cierto control centralizado, también recogen la gestión y evaluación descentralizada de las convocatorias.

4) Promueven los proyectos con "valor añadido", es decir, con aportaciones complementarias a las de las iniciativas territoriales más restringidas, es decir, permiten logros que sólo con las políticas y recursos territoriales no podrían alcanzarse.

5) Aportan la "dimensión europea" que, como cualquier dimensión que favorezca trascender el etnocentrismo, la multiculturalidad, la creación de redes y grupos con una mirada diferente pero complementaria de los problemas, supone un logro en sí mismo de las convocatorias de investigación: es otro valor añadido.

Todos estos elementos de las convocatorias europeas, pueden servir de valiosos referente para la negociación de una política científica en materia educativa en el estado español, que sin restar autonomía o competencias a las comunidades, permita hablar de una comunidad más amplia del conocimiento y la indagación sobre problemas educativos comunes, competencia que muy bien podría desarrollar un organismo como el CIDE. 


\section{Referencias bibliográficas y normativas utilizadas}

\section{Programa Sócrates}

DOCE L28/01, 3/2/00. Decisión 253/2000/CE del Parlamento Europeo y del Consejo, de 24 de Enero de 2000, por la que se establece la segunda fase del programa de acción comunitario en materia de educación Sócrates (Base jurídica).

DOCE C 121/09, 29/4/00. Convocatoria de propuestas de acciones generales de observación y análisis (Acción 6.1.2 del programa Sócrates) (Fecha límite: 31/7/00).

DOCE C 127/09, 5/5/00. Convocatoria de cursos para el catalogo Comenius (Fecha límite: 30/6/00).

DOCE C 159/07, 8/6/00. Convocatoria de manifestaciones de interés de posibles candidatos para el programa Sócrates, acción 1 de Lingua: Promoción del aprendizaje de lenguas (Fecha límite: $31 / 12 / 02)$.

DOCE C 275/11, 27/9/00. Programa de acción comunitario en materia de educación Sócrates. Convocatoria de propuestas 2001 (Fecha límite: 1/8/01).

BOE 39/5547, 14/2/01. Orden de 2 de febrero de 2001 por la que se convocan las ayudas correspondientes a las acciones descentralizadas de la segunda fase del programa Sócrates de la Unión Europea (Fecha límite: 20/2/01).

DOCE C 99/06, 29/3/01. Convocatoria de propuestas sobre las acciones generales de observación y análisis (Acción 6.1.2 del programa Sócrates) (Fecha límite: 1/6/01).

DOCE C 103/07, 3/4/01. Convocatoria de cursos ara el catálogo Comenius y Grundtvig (Programa Sócrates) (Fecha límite: 31/5/01).

DOCE C 177/05, 22/6/01. Convocatoria de propuestas (DG EAC 30/01). Acciones conjuntas. Programas Sócrates, Leonardo Da Vinci U Juventud (Fecha límite: 31/8/01).

$\operatorname{COM}(2001) 75$ final, 12/2/01. Informe final de la Comisión sobre la aplicación del programa Sócrates 1995-1999 (Documento).

DOCE C 191/10, 7/7/01. Convocatoria general de propuestas 2002 (EAC 29/01). Programa de acción comunitario en materia de educación Sócrates (la fecha límite para algunas acciones es el 1 de noviembre de 2001) (Fecha límite: 1/10/02).

\section{Programa Leonardo da Vinci}

DOCE L146/33, 11/6/99. Decisión 1999/382/CE, de 26 de abril de 1999, por la que se establece la segunda fase del programa de acción comunitario en materia de formación profesional Leonardo da Vinci (Base jurídica).

DOCE C 23/08, 27/1/00. Convocatoria de propuestas (2000, 2001 y 2002). (Fecha límite: 19/1/02) (Convocatoria).

DOCE C 1/06, 4/1/01. Convocatoria de candidaturas para la constitución de listas de expertos encargados de evaluar las propuestas recibidas en el marco del programa de acción comunitario en materia de formación profesional Leonardo da Vinci (Fecha límite: 13/3/01) (Convocatoria).

DOCE C 177/05, 22/6/01. Convocatoria de propuestas (DG EAC 30/01). Acciones conjuntas. Programas Sócrates, Leonardo da Vinci U Juventud (Fecha límite: 31/8/01) (Convocatoria).

$\operatorname{COM}(2000) 863$ final, 22/12/00. Informe final sobre la aplicación de la primera fase del programa de acción comunitario Leonardo da Vinci (1995-1999) (Documento). 


\section{Programa Cultura 2000}

DOCE L 63/01, 10/3/00. Decisión 508/2000/CE por la que se establece el programa Cultura 2000 (Base jurídica).

DOCE C 163/03, 10/6/99. Convocatoria de propuestas para acciones experimentales con miras al programa marco en favor de la cultura.

DOCE C 210/10, 22/7/00. Anuncio de convocatoria de manifestaciones de interés DG EAC/21/2000 relativa a prestaciones de servicios en el ámbito de la educación, la formación profesional, la cultura, la política audiovisual, el deporte, la ciudadanía y la juventud (Fecha límite: 31/1/03).

DOCE C 224/07, 5/8/00. Concurso para el establecimiento de un premio de la Unión Europea de arquitectura contemporánea (Fecha límite: 22/9/00).

DOCE C 21/08, 24/1/01. Apoyo de la Unión Europea a la cultura: Programa Cultura 2000. Aplicación del programa en el año 2001 y convocatoria de propuestas (Fecha límite: 15/5/01).

DOCE C 230/03, 15/8/01. Convocatoria de propuestas para el 2002 (Fecha límite: 30/11/01).

\section{Programa Media Formación}

DOCE L 26/01, 27/1/01. Decisión 163/2001/CE relativa a la ejecución de un programa de formación para los profesionales de la industria europea de programas audiovisuales (MEDIA-formación) (2001-2005) (Base jurídica).

DOCE C 63/08, 28/2/01. Realización de un programa de formación para los profesionales de la industria europea de programas audiovisuales (MEDIA-formación, 2001-2005). Anuncio de convocatoria de propuestas 12-2001 (Fecha límite: 25/4/01) (Convocatoria).

\section{Programa Alfa}

DOCE L 52/01, 27/2/92. Reglamento 443/92 (Base jurídica).

DOCE C 42/08, 15/2/00. Convocatoria de presentación de candidaturas 2000 (Fecha límite: 30/10/00) (Convocatoria).

DOCE C 102/14, 31/3/01. Programa ALFA: Convocatoria de propuestas; sistema de presentación continua (Fecha límite: 30/10/01) (Convocatoria).

COM (2001) 385 final, 18/7/01. Comunicación de la Comisión al Parlamente Europeo y al Consejo relativa al refuerzo de la cooperación con terceros países en materia de enseñanza superior.

ALFA (América Latina Formación Académica). Guía del Programa y del Candidato. Comisión Europea. Dirección América Latina.

\section{Programa Equal}

DOCE C 127/02, 5/5/00. Comunicación de la Comisión a los Estados miembros por la que se establecen las orientaciones relativas a la iniciativa comunitaria EQUAL, al respecto de la cooperación transnacional para promocionar nuevos métodos de lucha contra las discriminaciones y desigualdades de toda clase en relación con el mercado de trabajo (Base jurídica).

BOE 92/7468, 17/4/01. Resolución de 22 de marzo de 2001, de la Unidad Administradora del Fondo Social Europeo, por la que se convocan las ayudas del Fondo Social Europeo correspondiente a la Iniciativa comunitaria EQUAL en España (Fecha límite: 17/6/01) (Convocatoria). 
Comunidades Europeas (2000). Equal. Nuevas formas de combatir la discriminación y la desigualdad en el ámbito del empleo. Luxemburgo: Oficina de Publicaciones Oficiales de las Comunidades Europeas.

Programa de la Iniciativa Comunitaria EQUAL. Complemento de Programa, aprobado por el Comité de Seguimiento el 25/4/01.

\section{Programa Eumedis}

DOCE L 189/01, 30/7/96. Reglamento 1488/96 (programa MEDA) (Base jurídica).

DOCE C 88/09, 25/3/00. Convocatoria 2000 SCRE/110979/C/G (Fecha límite: 22/1/01) (Convocatoria).

\section{Programa Connect}

DOCE C 133/12, 13/5/99. Convocatoria de propuestas 1999.

DOCE C 163/04, 10/6/99. Convocatoria de propuestas para acciones preparatorias basadas en la sinergia entre la cultura y los ámbitos de la educación y la formación, que podrán incluir aspectos de investigación y nuevas tecnologías.

\section{Programa e-learning}

COM (2001) 172 final, 28/3/01. Comunicación sobre el Plan de acción eLearning, concebir la educación del futuro.

DOCE C 204/02, 20/7/01. Resolución de 13 de junio de 2001 relativa al eLearning.

CONVOCATORIA DOCE C 166/17, 9/6/01. Convocatoria de propuestas. Acciones preparatorias e innovadoras. Plan de Acción eLearning. DG EAC/25/01 (Fecha límite: 16/11/01).

\section{Programa Daphne}

DOCE L 34/01, 9/2/00. Decisión 293/2000/CE por la que se aprueba un programa de acción comunitario sobre medidas preventivas destinadas a combatir la violencia ejercida sobre los niños, los adolescentes y las mujeres (2000-2003).

DOCE C 73/09, 14/3/00. Convocatoria de propuestas para 2000 (Fecha límite: 2/6/00).

DOCE C 83/11, 14/3/01. Medidas preventivas destinadas a combatir la violencia ejercida sobre los niños, los adolescentes y las mujeres Convocatoria de propuestas para 2001 (Fecha límite: 28/5/01).

\section{Programa Marco}

Programa TSER: Incremento del Potencial humano de investigación y mejora de la base de conocimientos socioeconómicos

DOCE L 64/105, 12/3/99. Decisión 1999/173/CE por la que se aprueba un programa específico de investigación, demostración y desarrollo tecnológicos para el incremento del potencial humano de investigación y de la base de conocimientos socioeconómicos (1998-2002). 
DOCE C 361/09, 15/12/00. Convocatoria de propuestas de acciones indirectas de IDT para el programa específico de investigación, desarrollo tecnológico y demostración para incrementar el potencial humano de investigación y la base de conocimientos socioeconómicos. Premios de investigación. Premio Arquímedes. Referencia de la convocatoria: IHP-ARP-00-1 (Fecha límite: 31/7/01).

DOCE C 13/08, 16/1/01. Convocatoria de propuestas de acciones indirectas de IDT. Apoyo a la elaboración de políticas científicas y tecnológicas europeas. Base común de indicadores sobre ciencia, tecnología e innovación. Referencia de la convocatoria: IHP-CBSTII-01-1 (Fecha límite: $15 / 10 / 01)$.

DOCE C 33/05, 1/2/01. Convocatoria de propuestas de acciones indirectas de IDT para el programa específico de investigación, desarrollo tecnológico y demostración sobre el Incremento del potencial humano de investigación y de la base de conocimientos socioeconómicos, que sirve de apoyo al desarrollo de las políticas científicas y tecnológicas en Europa Análisis estratégico de cuestiones políticas específicas. Referencia de la convocatoria: IHP-STRATA-2001-1 (Fecha límite: 17/12/01).

DOCE C 107/14, 7/4/01. Convocatoria de propuestas para acciones indirectas de IDT en el marco del programa específico de investigación, desarrollo tecnológico y demostración sobre incrementar el potencial humano de investigación y la base de conocimientos socioeconómicos. Sensibilización pública sobre ciencia y tecnología. Referencia de la convocatoria: IHP-RPA-01-1 (Fecha límite: 7/7/01).

DOCE C 206/07, 24/7/01. Convocatoria de propuestas de acciones indirectas de IDT con arreglo al programa específico de investigación, demostración y desarrollo tecnológico para el Incremento del potencial humano de investigación y de la base de conocimientos socioeconómicos. Acción clave Mejora de la base de conocimientos socioeconómicos. Referencia de la convocatoria: IHPKA1-2001-1 (Fecha límite: 15/1/02).

DOCE C 246/04, 4/9/01. Convocatoria de propuestas de acciones indirectas de IDT para el programa específico de investigación, desarrollo tecnológico y demostración sobre incremento del potencial humano de investigación y de la base de conocimientos socioeconómicos. Premios de investigación. Premio Arquímedes. Referencia de la convocatoria: IHP-ARP-01-1 (Fecha límite: 15/3/02).

\section{Programa IST: Tecnologías de la sociedad de la información}

IST Work Programme 2001. (Programa de trabajo 2001. Tecnologías de la Sociedad de la Información. Un programa de investigación, desarrollo tecnológico y demostración del V Programa Marco). Documento .pdf.

IST Work Programme 2000. Documento .pdf.

Procedimientos de evaluación para el programa "La sociedad de la información fácilmente accesible a los usuarios". Programa IST. Anexo I. Documento .pdf.

\section{Programa Marco}

COM(2001) 282 final, 30/5/01. El Programa Marco y el Espacio Europeo de la Investigación: aplicación del artículo 169 y conexión en red de los programas nacionales.

$\operatorname{COM}(2001) 279$ final, 30/5/01. Propuesta de Decisión relativa a los programas específicos a través de los cuales se ejecuta el Programa Marco de la Comunidad Europea 2002-2006 de acciones de investigación, desarrollo tecnológico y demostración. 
$\operatorname{COM}(2001) 331$ final, 20/6/01. Una estrategia de movilidad para el Espacio Europeo de Investigación.

$\operatorname{COM}(2001) 346$ final, 25/6/01. La dimensión internacional del Espacio Europeo de Investigación. COM(2001) 549 final, 3/10/01. La dimensión regional del Espacio Europeo de la Investigación.

\section{Varios}

Mec (1989). Plan de Investigación Educativa y de Formación del Profesorado. Madrid: MEC.

Veláz de Medrano, C. (1997). "Los criterios de valoración de la calidad de la investigación educativa desde la doble perspectiva de los investigadores y de los organismos públicos que la promueven". Bordón, Vol. 49, n 4, pp. 457-477.

\section{Webs consultadas}

\section{Programa Sócrates}

http://www.mec.es/sgpe/socrates/ Servicio de Información Agencia Nacional Sócrates (excepto Erasmus).

http://www.mec.es/sgpe/socrates/convocatorias.htm Convocatorias Sócrates en vigor.

http://europa.eu.int/comm/education/socrates.html Home page del programa.

\section{Programa Leonardo da Vinci}

http://europa.eu.int/comm/education/leonardo2-en.html Home page del Programa.

http://www.mec.es/fp/leonardo Home page del programa en castellano.

\section{Programa Cultura 2000}

www.europa.eu.int/comm/culture/decision_es.pdf Decisión n ${ }^{0}$ 508/2000/CE Del Parlamento Europeo y del Consejo de 14 de febrero de 2000 por la que se establece el programa "Cultura 2000". http://europa.eu.int/comm/culture/index-en.html Home page del programa.

http://www.mcu.es/cooperacion/pcc/p_pcc.html Home page en castellano.

\section{Programa Media Formación}

http://europa.eu.int/comm/dg10/avpolicy/media/index-fr.html.

http://europa.eu.int/comm/avpolicy/media/index_en.html Home page del programa.

http://www.mediadeskspain.com Home page en castellano.

\section{Programa Alfa}

http://www.alfa-program.com/ Home page del programa. 


\section{Programa Equal}

http://europa.eu.int/comm/equal Home page del programa.

http://www.mtas.es/uafse/ Home page en castellano.

\section{Programa Eumedis}

http://www.ispo.cec.be/eumedis/ Home page del programa.

\section{Programa Connect}

www.europa.eu.int/comm/education/connect/selection.html Connect Iniciative.

www.europa.eu.int/comm/education/connect/123-1es.pdf Convocatoria de Propuestas iniciativas Connect DG XXII/10/99.

\section{Programa e-learning}

http://europa.eu.int/comm/education/elearning/index.html Home Page.

http://europa.eu.int/comm/education/elearning/what.htm Novedades.

http://europa.eu.int/comm/education/elearning/call.html Convocatoria anual.

\section{Programa Daphne}

http://europa.eu.int/comm/justice home/project/daphne/es Home page del programa.

http://europa.eu.int/comm/justice home/project/daphne/pdf/guidelines2001_es.pdf Guía y consejos de realización de proyectos.

\section{Programa Marco}

\section{General}

www.europa.eu.int/eur-lex/es/lif/dat/1999es 399Do182.html. Decisión no 182/1999/CE del Parlamento Europeo y del Consejo de 22 de Diciembre de 1998 relativa al quinto programa marco de la Comunidad Europea para acciones de investigación, demostración y desarrollos tecnológicos (1998-2002). DO n ${ }^{\circ}$ L 026 de 01/02/1999.

http://www.cordis.lu/fp5/home.html Fifth Framework Programme.

www.europa.eu.int/comm/research/guide/pdf/participation en.pdf Fifth Framework Programme guide.

www.cordis.lu/fp5/scr/struct.htm Programme Structure and Content.

www.cordis.lu/fp5/src/meas.htm Types of actions supported.

www.cordis.lu/fp5/src/criteria.htm Common set of criteria.

www.cordis.lu/fp5/monitoring/home.html Monitoring, evaluation \& assessment activities. 
www.cordis.lu/fp5/src/evalman.htm Evaluación del Quinto Programa Marco.

http://europa.eu.int/comm/research/index.html Investigación.

Programa TSER: Incremento del Potencial humano de investigación

$y$ de la base de conocimientos socioeconómicos

www.europa.eu.int/eur-lex/es/lif/dat/1999/es 399Do173.html Programa específico de investigación, demostración y desarrollo tecnológico para el incremento del Potencial humano de investigación y de la base de conocimientos socioeconómicos (1998-2002. DO. nº L 064 de 12/03/1999.

www.cordis.lu/tser/home.html Targeted Socio-economic Research. European Comisión-DG XII G. ftp://ftp.cordis.lu/pub/improving/docs/g_wp_es_199903.pdf Programa de Trabajo.

Diciembre 1999.

www.cordis.lu/fp5/src/evalman.htm Evaluación. Anexo N.

http://www.europa.eu.int/comm/research/faq.html Preguntas más frecuentes.

http://sost.cdti.es/proceso.htm Proceso de Preparación de propuestas.

http://www.cicyt.es/sost/programa71.htm.

Programa IST: Tecnologías de la sociedad de la información

http://www.cordis.lu/ist/part-docs.htm\#pg1 Información sobre el programa IST.

http://www.cordis.lu/ist/socioeconomic/home.html The socio-economic dimension of the IST programme.

www.cordis.lu/ist Programa IST.

www.cordis.lu/ist/work programme.htm Programa de Trabajo.

ftp://ftp.cordis.lu/pub/ist/docs/b wp es 2001001.pdf.

http://www.cordis.lu/pfp5/src/t-2.htm.

\section{Propuesta del VI Programa Marco}

ftp://ftp.cordis.lu/pub/ist/docs/fp6finalversion.pdf Documento legal.

http://europa.eu.int/comm/research/area en.htm.

http://www.europa.eu.int/comm/research/nfp.html Home page.

http://www.europa.eu.int/comm/dgs/research/selectio.html Staff Evaluador.

www.cordis.lu/ist/fp6/fp6consult.htm Programa TSI.

http://europa.eu.int/comm/research/index_es.html Home.

http://www.cordis.lu/rtd2002/home.html últimas novedades VIPM.

http://www.europarl.eu.int/meetdocs/committees/itre/20011009/449238en.pdf.

\section{Varios Evaluación}

$\underline{\text { www.cordis.lu/ist/part-docs.htm\#pg1 }}$ 


\section{Varios}

http://europa.eu.int/comm/secretariat_general/sgc/info_subv/commun/shortvad-es.pdf Vademecun de la Gestión de ayudas para solicitantes y beneficiarios de ayuda Europea.

www.comadrid.es/webdgpe Programas Internacionales.

www.europa.eu.int/comm/education/progr.html Programas Europeos de educación.

www.biblioteca.uca.es/sbuca/bibedu/enlaces.htm\#// Base de datos sobre educación.

www.cordis.lu/es/home.html Página principal del servidor Cordis.

www.cordis.lu/cerif The common European Research Information Format.

http://www.guiafc.com Guía de financiación comunitaria.

Fecha de recepción: 25-11-03

Fecha de revisión: 03-12-04

Fecha de aceptación: 20-04-05 